\title{
A fungus boosts dengue virus in the mosquito gut
}

Trypsin activity
was found
to decrease
significantly
in mosquito
midguts and
in vitro after
exposure to
the fungal
secretome

The mosquito Aedes aegypti is the principal vector of dengue virus (DENV), the cause of the dengue fever epidemic in tropical and sub-tropical regions worldwide. Transmission of DENV in the human population relies on the successful completion of its infection cycle in the mosquito; after ingestion of blood from an infected individual, DENV replicates in the mosquito midgut and disseminates to the salivary glands where it can be transmitted to new hosts through a mosquito bite. The gut of the mosquito is colonized with bacteria that influence DENV infection; however, whether

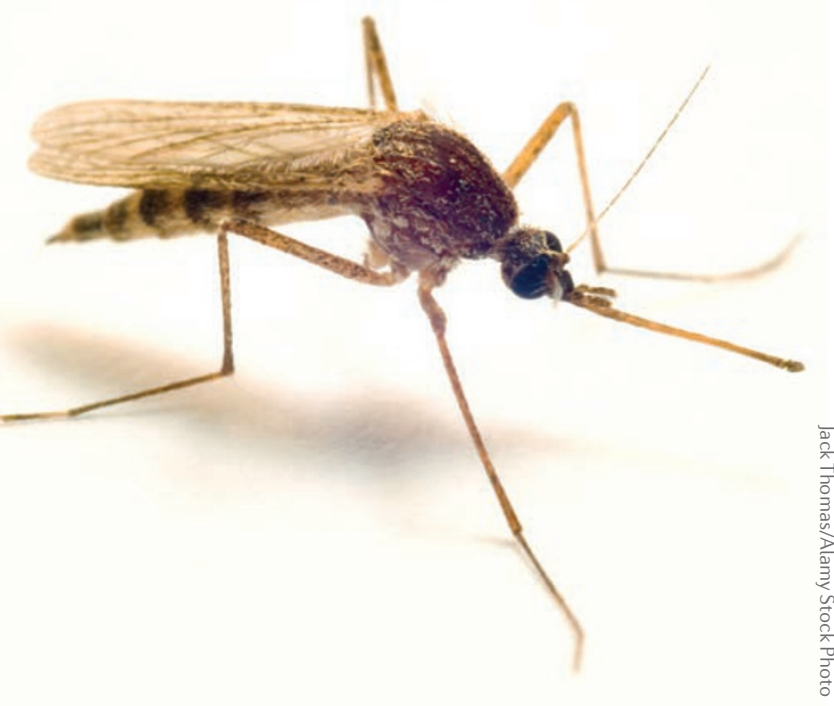

gut-associated fungi have an effect on DENV infection remains unknown. Dimopoulos and colleagues now find that a gut-associated fungus alters the gut physiology of $A$. aegypti to increase susceptibility to DENV.

The authors isolated a Talaromyces species of fungus from the midgut of $A$. aegypti mosquitoes that were collected from the DENVendemic region of Maunabo, Puerto Rico (they hence named the fungus Talaromyces sp. PR). They confirmed that the Talaromyces sp. was able to colonize the mosquito midgut and that its presence did not affect the lifespan of the mosquito. To test whether Talaromyces sp. PR could modulate susceptibility to DENV infection, the authors introduced fungal spores to the diet of two A. aegypti strains prior to a DENVinfected blood meal. Spore ingestion significantly increased DENV titres in both strains, suggesting that Talaromyces sp. PR augments DENV replication in the mosquito midgut.

As Talaromyces spp. produce and secrete a range of secondary metabolites and proteins, the authors tested whether the effects were due to the presence of whole live fungi or a secreted factor. The ingestion of a sucrose suspension of filtered Talaromyces sp. PR culture medium that lacked either spores or mycelia was sufficient to augment
DENV replication. Importantly, the Talaromyces sp. PR secretome did not affect bacteria in the midgut or influence DENV titres in antibiotic-treated mosquitoes.

To identify the underlying mechanism for these observations, the authors performed a genome-wide, microarray-based transcriptome comparison between mosquitoes that were exposed to the fungal secretome and those that were not and found that a significant proportion of genes that are involved in gut digestion were down-regulated in the mosquitoes that were exposed, and 22\% of the total down-regulated genes encoded trypsins. Trypsin activity was found to decrease significantly in mosquito midguts and in vitro after exposure to the fungal secretome, and RNAi of trypsin genes in Talaromyces sp. PR significantly increased DENV replication.

In this series of experiments, the authors show that one or more factors that are secreted by Talaromyces sp. PR modulate typsin expression and activity in the A. aegypti midgut, which augments viral replication and may facilitate DENV transmission in human populations.

Ashley York

ORIGINAL ARTICLE Angleró-Rodríguez, Y. I. et al. An Aedes aegypti-associated fungus increases susceptibility to dengue virus by modulating gut trypsin activity. eLife 6, e28844 (2017) 